\title{
Leveraging allelic heterogeneity to increase power of association
}

\section{testing}

Farhad Hormozdiari ${ }^{1,2}$, Junghyun Jung ${ }^{3}$, Eleazar Eskin*4,5, and Jong Wha J. Joo*6

${ }^{1}$ Department of Epidemiology, Harvard T.H. Chan School of Public Health, Boston, MA

$$
\text { 02115, USA }
$$

${ }^{2}$ Program in Medical and Population Genetics, Broad Institute of MIT and Harvard, Cambridge, Massachusetts, USA

${ }^{3}$ Department of Life Science, Dongguk University-Seoul, 04620 Seoul, South Korea

${ }^{4}$ Department of Computer Science, University of California, Los Angeles, California 90095

${ }^{5}$ Department of Human Genetics, University of California, Los Angeles, CA 90095, USA

${ }^{6}$ Department of Computer Science and Engineering, Dongguk University-Seoul, 04620

Seoul, South Korea

hormozdiari@hsph.harvard.edu,swordbread@dongguk.edu,eeskin@cs.ucla.edu, jwjjoo@dongguk.edu

${ }^{*}$ Corresponding author: eeskin@cs.ucla.edu, jwjjoo@dongguk.edu 


\begin{abstract}
The standard genome-wide association studies (GWAS) detects an association between a single variant and a phenotype of interest. Recently, several studies reported that at many risk loci, there may exist multiple causal variants. For a locus with multiple causal variants with small effect sizes, the standard association test is underpowered to detect the associations. Alternatively, an approach considering effects of multiple variants simultaneously may increase statistical power by leveraging effects of multiple causal variants. In this paper, we propose a new statistical method, Model-based Association test Reflecting causal Status (MARS), that tries to find an association between variants in risk loci and a phenotype, considering the causal status of the variants. One of the main advantages of MARS is that it only requires the existing summary statistics to detect associated risk loci. Thus, MARS is applicable to any association study with summary statistics, even though individual level data is not available for the study. Utilizing extensive simulated data sets, we show that MARS increases the power of detecting true associated risk loci compared to previous approaches that consider multiple variants, while robustly controls the type I error. Applied to data of 44 tissues provided by the GenotypeTissue Expression (GTEx) consortium, we show that MARS identifies more eGenes compared to previous approaches in most of the tissues; e.g. MARS identified $16 \%$ more eGenes than the ones reported by the GTEx consortium. Moreover, applied to Northern Finland Birth Cohort (NFBC) data, we demonstrate that MARS effectively identifies association loci with improved power ( $56 \%$ of more loci found by MARS) in GWAS studies compared to the standard association test.
\end{abstract}




\section{Introduction}

Over the past decade, genome-wide association studies (GWAS) have successfully identified many variants significantly associated with diseases and complex traits. Unfortunately, those variants explain an extremely small proportion of phenotypic variation [1, 2] and there are many more variants with even smaller effects that we are yet to identify [1, 3/5]. Detecting all loci that harbor associated risk loci can help elucidate the biological mechanisms of diseases and complex traits. All biological follow up studies are performed on loci that harbor at least one significant variant. The standard association test used in GWAS examines one variant at a time to identify associated variants, we refer to this method as the univariate test.

It has been shown in previous works that many loci in the genome harbor more than one causal variant for a given disease or a trait [6-15]. In addition, recent works have demonstrated widespread allelic heterogeneity in expression quantitative trait loci (eQTLs) and complex traits [16, 17]. For a locus containing multiple causal variants with small effect sizes, the univariate test may be underpowered. Alternatively, an approach considering effects of multiple causal variants simultaneously may increase statistical power to detect signals for the locus by aggregating the effects of causal variants.

In this paper, we propose a new model-based method for identifying an association between multiple variants in a locus and a trait that we call Model-based Association test Reflecting causal Status (MARS). Our approach builds upon recent progress in fine mapping approaches that try to identify causal variants in a locus. Causal variants are the variants that are responsible for the association signal at a locus. However, at each locus, there are often tens to hundreds of variants tightly linked (linkage disequilibrium, LD) to the reported associated single nucleotide polymorphism (SNP), therefore, the LD hinders the identification of causal variants at the risk locus. CAVIAR [13] is one of the recent fine mapping approaches that estimates the probability of each variant being causal, allowing an arbitrary number of causal variants by jointly modeling association statistics at all the variants. We extend the likelihood model of CAVIAR to explicitly incorporate LD structure of data utilizing MVN distribution conditional on causal status of the variants. We perform a Likelihood Ratio Test (LRT) that computes the likelihood ratio of a null model, where none of the variants are causal, and an alternative model, where at least one of the variants is causal. For the significance 
test, we apply an efficient re-sampling approach.

Our method does not require individual level data, which is often not provided in GWAS. MARS only requires summary statistics and LD of variants in a locus, which can be obtained from a reference dataset such as HapMap [18, 19] or 1000 Genome project [20], and reports a $p$-value that indicates the significance of the association between the locus and the corresponding trait. This approach is related to set-based association tests that examine an association between a set of variants and a trait [21-23]. MARS outperforms these previous methods because the underlying model, building upon the model of CAVIAR, explicitly models the joint distribution of the observed statistics given multiple signals of associations. Furthermore, MARS uses a significance level which corresponds to the standard GWAS significance level facilitating interpretation.

Applied to several simulated data sets, we show that MARS robustly controls type I error and improves statistical power compared to the univariate test as well as the widely utilized set-based association test, fastBAT (a fast and flexible set-Based Association Test using GWAS summary data) 24]. Applied to data of 44 tissues provided by the Genotype-Tissue Expression (GTEx) consortium [25, 26], MARS identifies more eGenes, which are genes with at least one variant in cis significantly associated, than the ones reported by the GTEx consortium in most of the tissues, e.g. MARS identified $29 \%$ more eGenes than the ones reported by the consortium for the Whole Blood data and $57 \%$ of the extra eGenes, which were identified by MARS but not detected by the consortium, were reported in studies elsewhere. In order to demonstrate the increased power of MARS on real data, we followed a strategy of applying MARS on an older data set and validated additionally discovered loci using current datasets that have higher statistical power because they are much larger. Applied to the 2009 Northern Finland Birth Cohort (NFBC) data, we show that MARS effectively identifies more association loci than the univariate test and show that many of the new loci have since been discovered in recent GWAS studies.

\section{Results}

\section{Overview of MARS}

Causal variants are variants that are responsible for the association signal at a locus. The ultimate goal of the standard association test, which examines an association between each variant and a 
trait, is to find causal variants. We refer this method as the univariate test. However, often there exist multiple causal variants with small effect sizes in a locus. For these cases, the univariate test may not detect those associations due to its low statistical power. Alternatively, we can examine an aggregated effect of multiple variants simultaneously on the trait to increase statistical power.

We developed a novel statistical method referred to as Model-based Association test Reflecting causal Status (MARS). MARS examines an association between a set of variants and a trait. MARS requires summary statistics estimated for variants (e.g. z-score) for a locus of interest and correlation structure, LD, between the variants, which can be readily obtained from a reference dataset. To test the association between a set of variants of a locus and a trait, MARS performs the Likelihood Ratio Test (LRT) to compute a test statistic, referred to as $L R T_{\text {score }}$. We consider likelihoods of two models; the likelihood of the null model $\left(L_{0}\right)$ and the likelihood of the alternative model $\left(L_{1}\right)$. The null model assumes that there is no causal variant to the trait and the alternative model assumes that there is at least one causal variant to the trait. Then we compute the $L R T_{\text {score }}$ as $L_{1} / L_{0}$. Let's say we are testing the association between $m$ number of variants and a trait. Given the observed summary statistics we can compute the $L R T_{\text {score }}$ as follows:

$$
L R T_{\text {score }}=\frac{\sum_{C \in \zeta} p(S \mid C) p(C)}{p\left(S \mid C_{0}\right) p\left(C_{0}\right)}
$$

Here, $S=\left[s_{1}, \cdots, s_{m}\right]^{\mathbf{T}}$ indicates summary statistics of $m$ variants and $C$ indicates the causal status of $m$ variants. $C$ is a binary vector of length $m$, where 0 indicates a variant is non-causal and 1 indicates a variant is causal. Specifically, $C_{0}$ indicates the causal status where none of the variants are causal and $\zeta$ is a set that contains all the possible casual statuses except for the $C_{0}$. Since there are $m$ number of variants, there are $2^{m}$ possible causal statuses.

To assess an association significance for a locus, we utilize re-sampling approach, where we sample null statistics from a MVN distribution with corresponding LD and estimate $L R T_{\text {scores }}$ for the null statistics, to generate a null panel of $L R T_{\text {scores }} ; R R T_{\text {scores }}^{N L L}$. From the null panel, we estimate the siginificance of $L R T_{\text {score }}^{D A T A}$ computed from the data. Figure 1 shows the basic overall process of MARS. The details and techniques to make this process computationally feasible for a big genomic data are described in the Materials and Methods. 


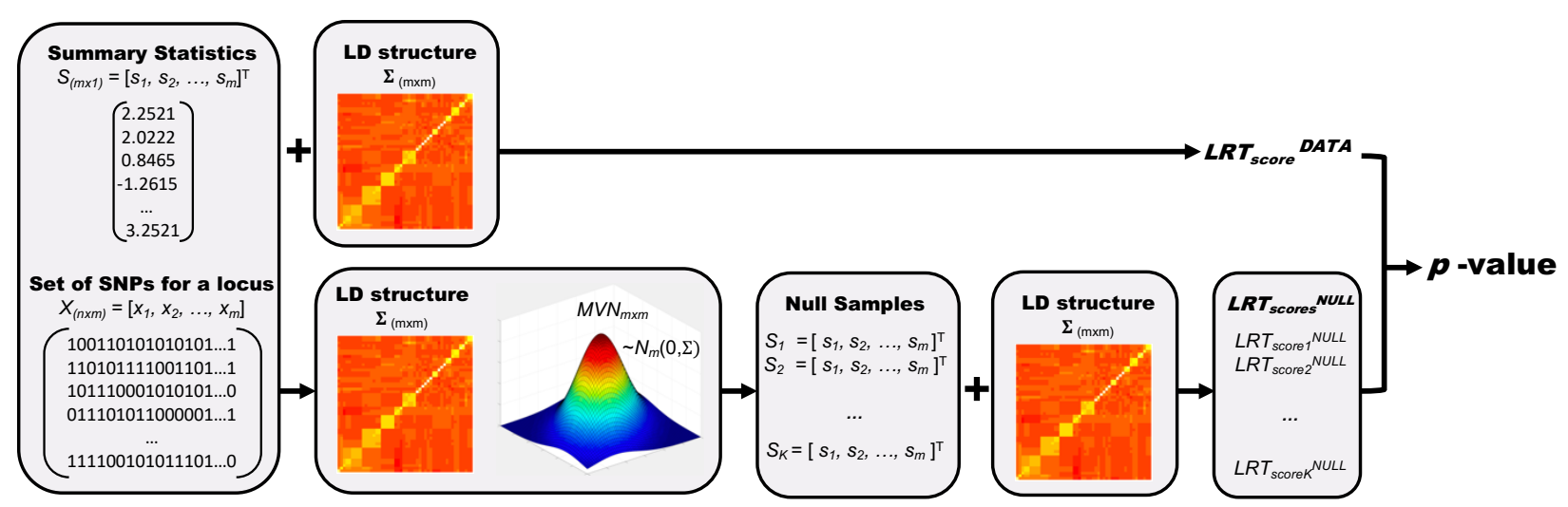

Figure 1: Overview of MARS In this figure we assume that we are testing an association between a locus of $m$ variants and a trait. The leftmost panel shows the input of MARS; $m$ number of summary statistics for the locus and $n \times m$ matrix, containing genotypes of $m$ SNPs for $n$ samples. The next two panels in the bottom show the re-sampling process that we sample null statistics $K$ times from a MVN distribution with a variance-covariance matrix of $\Sigma$, which contains LD of the genotypes $X$. The right most panel shows the process we estimate $L R T_{\text {scores }}$ for the null panel from which we compute a $p$-value of the data.

\section{MARS controls type I error while improves power in simulation studies}

We demonstrate that MARS controls type I errors through simulations of null panels utilizing the GTEx data as a starting point and consider the SNPs $\pm 1 \mathrm{Mb}$ around transcription start site (TSS) of 10 genes of Whole Blood data from the GTEx consortium [25, 26]. The half of the genes are randomly selected from genes reported as eGenes by the GTEx consortium and the other half of the genes are randomly selected from the rest of the genes, non-eGenes, of GTEx consortium [25, 26]. For each gene locus, we simulate $10^{8}$ number of null summary statistics according to the generative model described in the Materials and Methods, which uses the LD structure estimated from the genotypes of the SNPs in the locus and apply MARS to compute $L R T_{\text {scores }}$.

To show that MARS controls type I error, the false positive rates are estimated for different thresholds of $\alpha=0.01,0.05$, and 0.1 . The first half of the simulated data is used to compute a threshold of $L R T_{\text {scores }}$ for the corresponding $\alpha ; L R T_{\text {threshold }^{\alpha}}$ and the rest half of the simulated data is used to compute a quantile of $L R T_{\text {scores }}$ smaller than the $L R T_{\text {threshold }^{\alpha}}$. Figure 2 (a) shows that MARS robustly controls type I error for all the examined gene loci as the false positive rates for different gene loci are very close to the corresponding $\alpha=0.01,0.05$, and 0.1 , accordingly.

To show that MARS increases statistical power, we perform extensive simulation studies for various scenarios and compare the power of MARS with those of the univariate test. Here, we define the 
univariate test as a set-based association test that uses the maximum summary statistic among the SNPs in a locus we are testing (for the details see Materials and Methods). The same gene loci from the previous section are used for the test. We estimate power of each gene locus for cases with 2 causal variants implanted with different effect sizes of $\lambda=4,4.5,5,5.5$, and 6 . For a fair comparison of the powers between the univariate test and MARS, we utilize the standard GWAS $p$-value threshold of $5 \times 10^{-8}$. We simulate $10^{8}$ summary statistics under the null model of no effect to generate a null panel and $10^{8}$ summary statistics under the alternative model of effect size $\lambda$ for 2 causal variants to examine the power. From the null panel, we find a LRT threshold that corresponds to the $p$-value threshold of $5 \times 10^{-8}$. Then the power is estimated as a quantile of alternative cases that show $L R T_{\text {scores }}$ greater than the LRT threshold. For the detail, see Materials and Method. The percentage of power improvement is defined as (power of MARS power of the univariate test) $/($ power of the univariate test $) \times 100$. Figure 2 (b) shows that MARS increases statistical power compared to the univariate test. The extent of power improvements differs between the gene loci as the LD structures are different between the loci, but for all the cases, it is clear that the powers are improved over the univariate test. Depending on the effect size $\lambda$ implanted in the simulated data, the power improves from $5.2 \%$ to $41.18 \%$ in our experiments and as expected, the smaller the effect size is, the better MARS performs over the univariate test. The results do not show noticeable differences between loci of eGenes and loci of non-eGenes used for the simulations.

In addition, we examine the cases, where 3 causal variants, each with an effect size of $\lambda=4.5$, are implanted in the simulated data. As the number of causal variants increases from 2 to 3 , MARS shows bigger power improvement over the univariate test Figure 2 (c). The result shows that the more the number of causal variants exist in a locus, the better MARS performs over the univariate test.

Besides the univariate test, we compared MARS with one of the widely used set-based association test methods, fastBAT [24]. Because of the heavy I/O of fastBAT, we used $10^{5}$ simulations and a threshold of $10^{-5}$, which is large enough to evaluate and compare the methods. We compute the power of MARS and fastBAT for different effect sizes of $\lambda=1.5,2,2.5,3,3.5,4,4.5,5,5.5,6$ and show that MARS outperforms fastBAT for all the cases by improving power from $2 \%$ to $42 \%$ depending on the effect sizes in the experiments (Figure 2 (d)). 


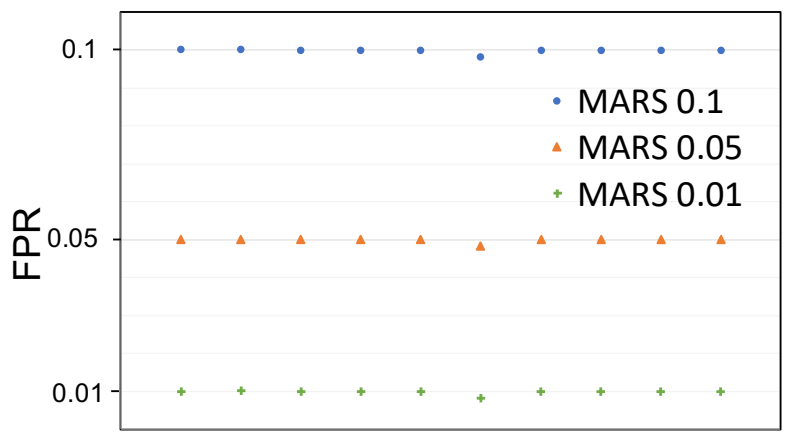

Gene locus

(a)

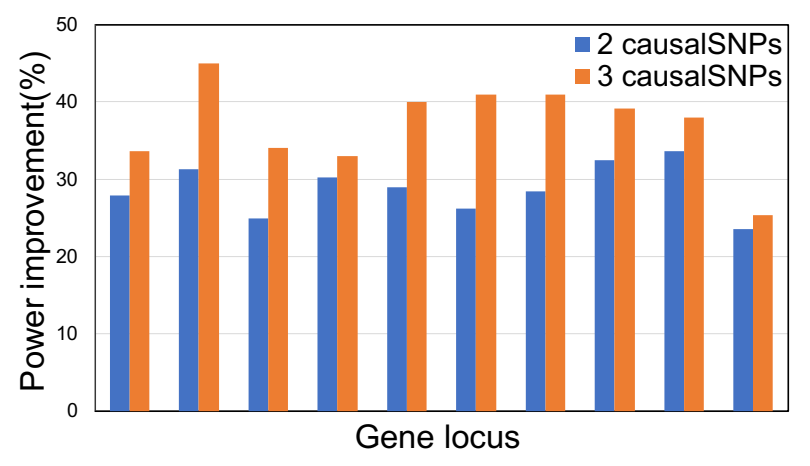

(c)

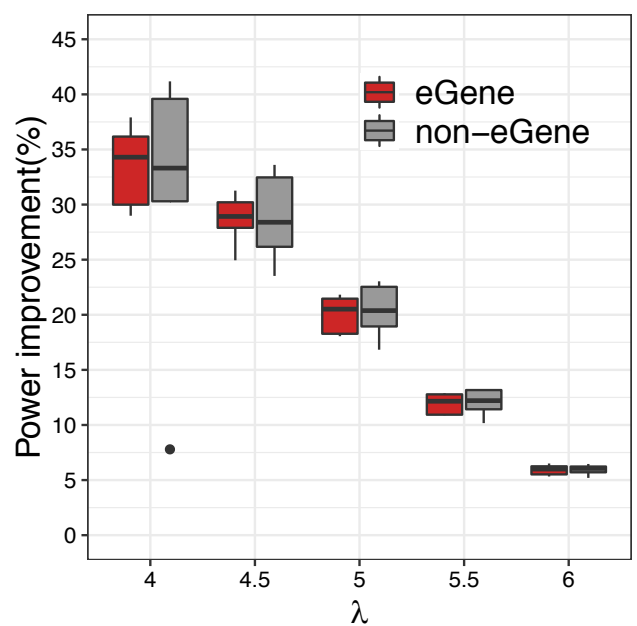

(b)

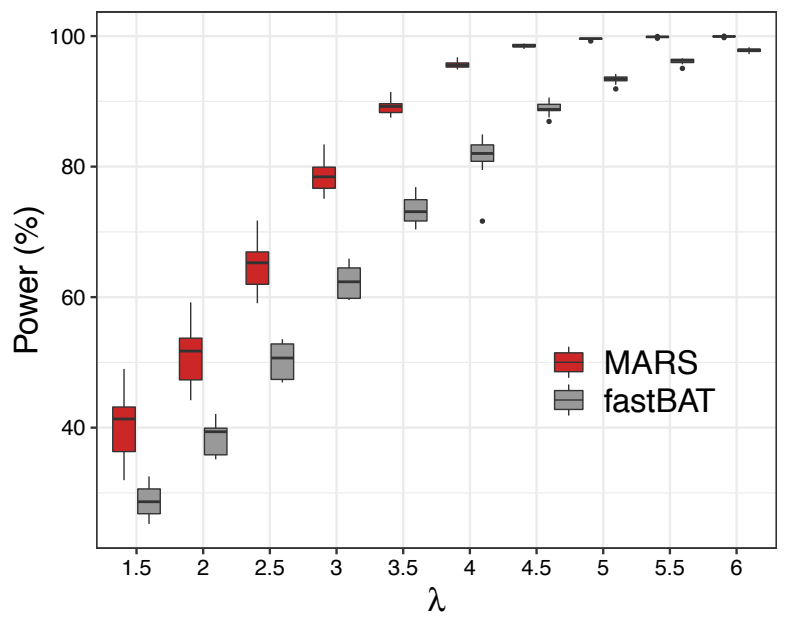

(d)

Figure 2: Comparison of eGenes identified by MARS and eGenes reported by GTEx consortium. (a) Plot shows that MARS controls type I error. The x-axis shows 10 different gene loci used for the test, where the first 5 gene loci are from eGenes that are reported by the GTEx consortium. The y-axis shows the false positive rates. The green plus, orange triangle, and blue circle show the false positive rates at $\alpha=0.01,0.05$, and 0.1 , accordingly. (b) A box plot showing the percentage of power improvement of MARS over the univariate test for different effect sizes of 2 causal SNPs exist in the data. The X-axis shows 5 different effect sizes of $\lambda=4,4.5,5,5.5$, and 6 used for the test. The $\mathrm{Y}$-axis shows the percentage of power improvement. The red bars and the black bars show power improvement when loci not reported and reported as eGenes by the GTEx consortium are used for the test, accordingly. (c) A box plot showing the power of MARS and fastBAT for different effect sizes. The X-axis shows effect sizes of $\lambda=1.5,2,2.5,3,3.5,4,4.5,5,5.5$, and 6 used for the test. The Y-axis shows the power in percentage. The red bars and the black bars show power of MARS and fastBAT, accordingly. 


\section{MARS detects novel eGenes in GTEx data}

Recently, a larger number of expression quantitative trait loci (eQTLs) studies have been reported. In particular, numerous cis-eQTLs, which are eQTLs that map to the approximate location of their gene-of-origin, have been identified. As part of this effort, GTEx consortium reported eGenes, which are genes with at least one cis-eQTL. We applied MARS to GTEx data to show that MARS can detect more eGenes than the ones reported by the GTEx consortium. Among the 44 tissues provided by the GTEx consortium, we first applied MARS to the Whole Blood data for the evaluation as the data contains the largest number of samples among all the tissues. To compare our results with GTEx's results, we use 10000 number of simulations because it is the number of simulations used by GTEx consortium to compute 'empirical p-values' to select eGenes. To identify eGenes for MARS, we set the threshold as the border of empirical $p$-value between eGenes and genes other than those eGenes, referred to as non-eGenes, reported by the GTEx consortium. Figure 3 shows a Venn diagram comparing the identifications of eGenes by MARS and the ones reported by the GTEx consortium. MARS identifies 2043 extra eGenes that are not reported by the consortium, while MARS misses only 98 eGenes that are reported by the consortium [25, 26]. MARS and the GTEx consortium detects 6686 common eGenes.

To verify that the eGenes identified by MARS are true associations, we compared those extra eGenes with those reported by other studies with larger sample sizes. Note that the results throughout the paper used data from GTEx version 6. Recently, GTEx version 7 has been published with more samples and improved technology in experiments. We expect more eGenes are detected in the newer version of the data as the power increases with the number of samples and etc. We compared the extra eGenes with the eGenes reported by GTEx version 7. In addition, we compared those extra eGenes with the eGenes reported by Framingham Heart Study (FHS) [27] that used the Whole Blood data on a large number of samples; 5257 samples. Figure 3 b shows a Venn diagram comparing identifications of eGenes of four studies, GTEx version 6, GTEx version 7, FHS, and MARS. Among the 2043 extra eGenes, 57\% of the genes (1160 genes) are reported in either GTEx version 7 or FHS; 763 genes are reported as eGenes in the GTEx version 7, 775 genes are reported as eGenes in FHS, and 378 genes are identified as eGenes by both of the studies, GTEx version 7 and FHS. Even with the older version of the data, MARS still finds more eGenes 
than GTEx version 7 and MARS is expected to identify even more eGenes if a data with larger number of samples is used in the further studies. Moreover, some of the genes among the 883 genes (Figure 3 b) that are identified only by MARS but not by any other studies, GTEx version 6, GTEx version 7, nor FHS, have biological evidences of being eGenes based on many literatures. Variants of $S P 140$ (ENSG00000079263) gene are known to be related to multiple sclerosis (MS) [28] and chronic lymphocytic leukemia [29]. Sille et al. have demonstrated that expression level of $S P 140$ is regulated by cis-eQTLs in lymphoblastoid cell lines [30]. Besides, the $S P 140$ protein levels are shown to be down-regulated by cis-acting mechanism in peripheral blood mononuclear cells (PBMCs) from MS patients [31]. HSPB8 (ENSG00000152137) has been recently identified as an eGene using PBMCs and the expression level of HSPB8 is known to be regulated by several SNPs [32]. Surfactant protein D encoded by SFTPD (ENSG00000133661) gene is known to be regulated by cis-acting manner in human blood [33], and CD83 (ENSG00000112149) has been recently identified as cis-eQTLs gene in CD19+ B lymphocyte [34. Additionally, in Supplementary Information 2, we thoroughly analyzed randomly selected 100 genes and compared their $p$-values of MARS, the univariate test, the ones reported by the GTEx consoritium to show that MARS identifies more eGenes with better $p$-values. These results show that MARS is capable of identifying novel eGenes that cannot be detected with the standard association test approaches. The list of 2048 extra eGenes and their identifications in GTEx version 7 and FHS is provided in the Supplementary Information 2.

One of the advantages of MARS is that once the null panel of $L R T_{\text {scores }}$ for each gene has been established, it can be applied to the gene in any other tissues. Utilizing the null panel of $L R T_{\text {scores }}$ estimated from the Whole Blood data of the GTEx consortium, we computed $p$-values of the genes in all 44 tissues of GTEx using their summary statistics and LD structures. Figure 4 shows that MARS identifies comparable or more eGenes than the univariate test as well as the ones reported by the GTEx consortium in all the tissues. As expected, the number of eGenes identified by the univariate test and those reported by the GTEx consortium are very close to each other in all of the tissues. The numbers of genes are different between tissues due to the sample size differences and etc., and we used only the genes common in each tissue and the Whole Blood data of the GTEx consortium because the null panel of $L R T_{\text {scores }}$ was estimated for genes in the Whole Blood data. Comparison of eGenes identified by MARS, the univariate test, and the GTEx consortium 


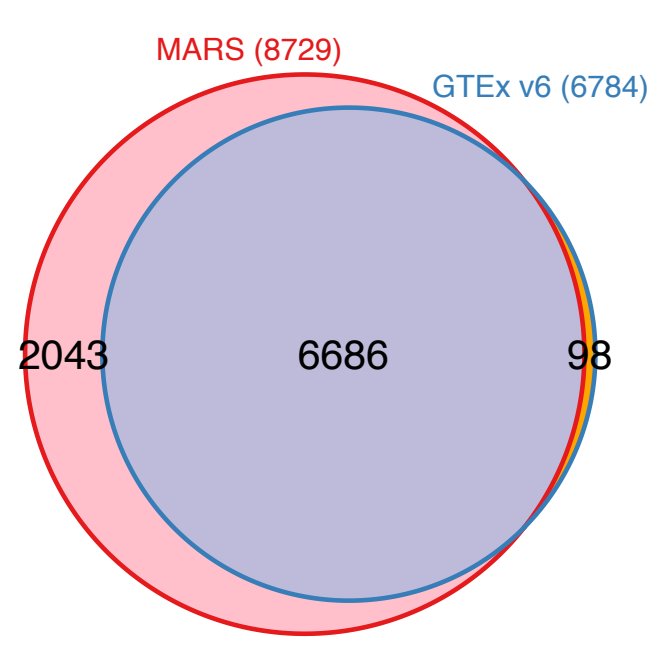

(a)

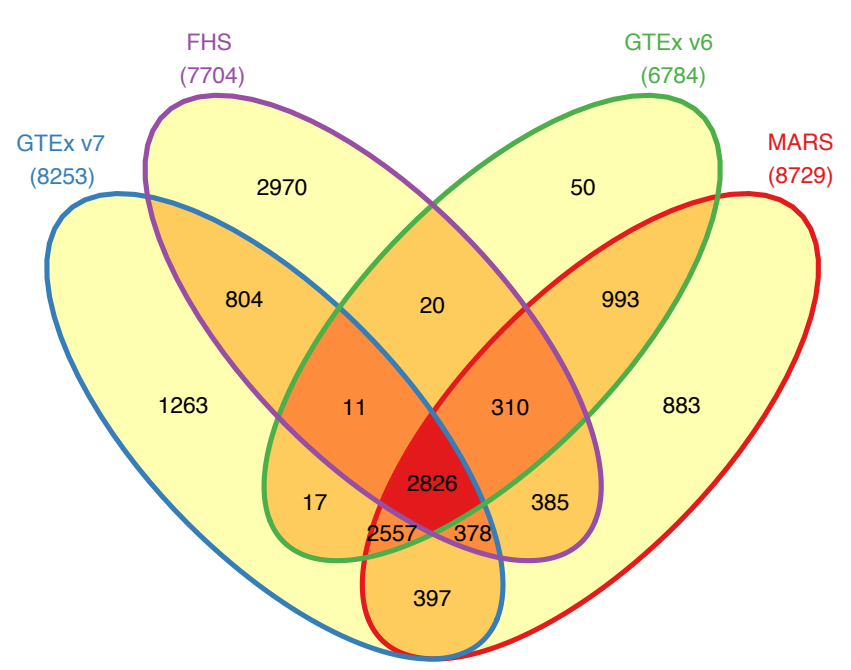

(b)

Figure 3: Comparison of eGenes identified by MARS and eGenes reported by GTEx consortium. (a) The red circle in the Venn diagram shows the eGenes identified by MARS and the blue circle in the Venn diagram shows the eGenes detected by GTEx version 6 . Whole blood data is used for the analysis. (b) Venn diagram comparing eGenes identified by GTEx version 6, GTEx version 7, FHS, and MARS. Whole blood data is used for all three of the studies. The blue, purple, green, and red circle shows the eGenes identified by GTEx version 7, FHS (Framingham Heart Study), GTEx version 7, and MARS, accordingly. Note that MARS used data from GTEx version 6 .

for each tissue is provided in the Supplementary Information 3. In addition, we compared eGenes identified by MARS, GTEx version 6, and GTEx version 7 and provide Venn diagrams as the one in the Figure 3 for all of the tissues (Supplementary Information 4).

\section{MARS detects more set-based associations in GWAS}

We show the effectiveness of our method on GWAS by applying MARS to the Northern Finland Birth Cohort (NFBC) data [35]. NFBC data consist of 10 traits collected from 5,327 individuals. The 10 traits are triglycerides (TG), high-density lipoproteins (HDL), low-density lipoproteins (LDL), glucose (GLU), insulin (INS), body mass index (BMI), C-reactive protein (CRP) as a measure of inflammation, systolic blood pressure (SBP), diastolic blood pressure (DBP), and height. For NFBC data we examined 51762 loci, where each locus is defined as $\pm 1 \mathrm{Mb}$ of TSS of genes provided by the GTEx consortium. 


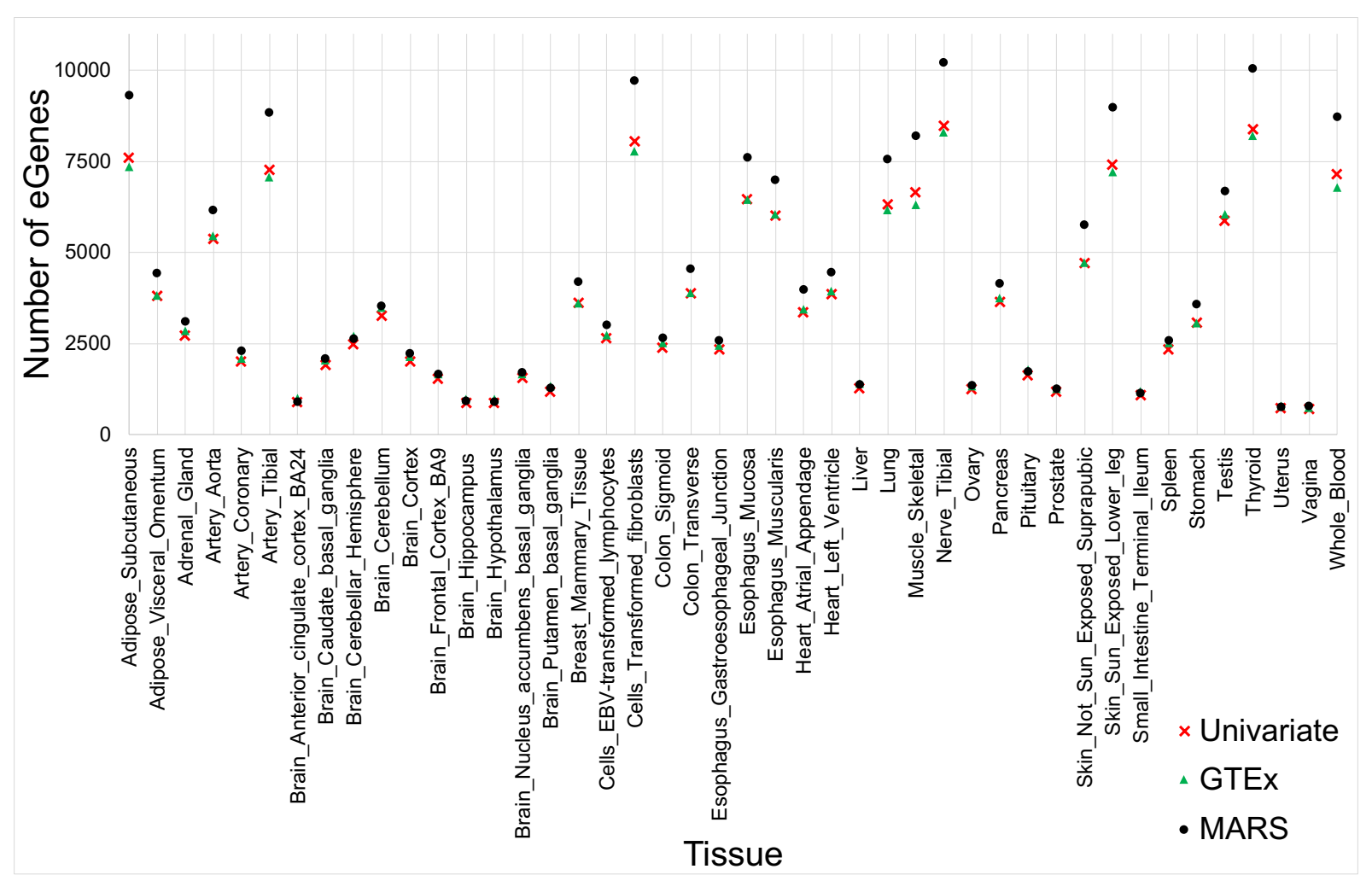

Figure 4: Number of eGenes identified by MARS, the univariate test, and those reported by the GTEx consortium. The x-axis shows the 44 tissues provided by the GTEx consortium and the y-axis shows the number of eGenes identified by each method. The black circle shows the number of eGenes identified by MARS, the red cross shows the number of eGenes identified by the univariate test, and the green triangle shows the number of eGenes reported by the GTEx consortium.

To apply the standard $p$-value threshold of $5 \times 10^{-8}$, MARS requires a lot of sampling. To reduce the running time, we apply the idea of importance sampling on MARS, which well approximates the $p$-value estimated from the original sampling approach, while reduces the number of sampling dramatically; from $10^{8}$ to $10^{4}$ (Supplementary Information 5). We refer to this efficient version of MARS as fastMARS. For the details, see Materials and Methods, section Fast and space efficient sampling for MARS. Figure 5 (a) shows that, for all the traits, fastMARS identifies more or comparable loci that are likely to be significantly associated with the traits. Significantly associated loci identified by fastMARS and the ones identified by the univariate test are listed in the Supplementary Information 6 and Venn diagrams comparing the identifications of fastMARS and the univariate test are provided in the Supplementary Information 7. 471 number of loci are identified only by fastMARS but not by the univariate test. To verify those extra loci, we searched the 
loci from other GWAS utilizing GWAS catalog [36]. As a result, several variants associated with 311 loci among the 471 extra loci were previously reported elsewhere in different studies [7, 3761. For example, rs6060369 locus associated with height was reported by large GWAS [44 46]. rs1800961 locus related to HDL was previously reported by large GWAS as well as meta-analysis GWAS [54 56, 60, 61]. rs6511720 locus related to LDL was discovered by several previous studies [40, 50, 51, 55, 56, 60]. A Venn diagram in Figure 5 (b) compares the number of loci identified by fastMARS and the univariate test as well as it shows the number of loci, for which at least one associated variant has been reported by GWAS catalog. The list of SNPs, the corresponding loci found by previous studies, and their detailed information including PubMed id and SNP position is provided in the Supplementary Information 8. Note that loci are defined based on the gene map of GTEx $( \pm 1 \mathrm{Mb}$ of TSS), thus some loci may overlap. For the details, see the Supplementary Information 8. These results demonstrate that fastMARS can efficiently identify novel associations in GWAS. 

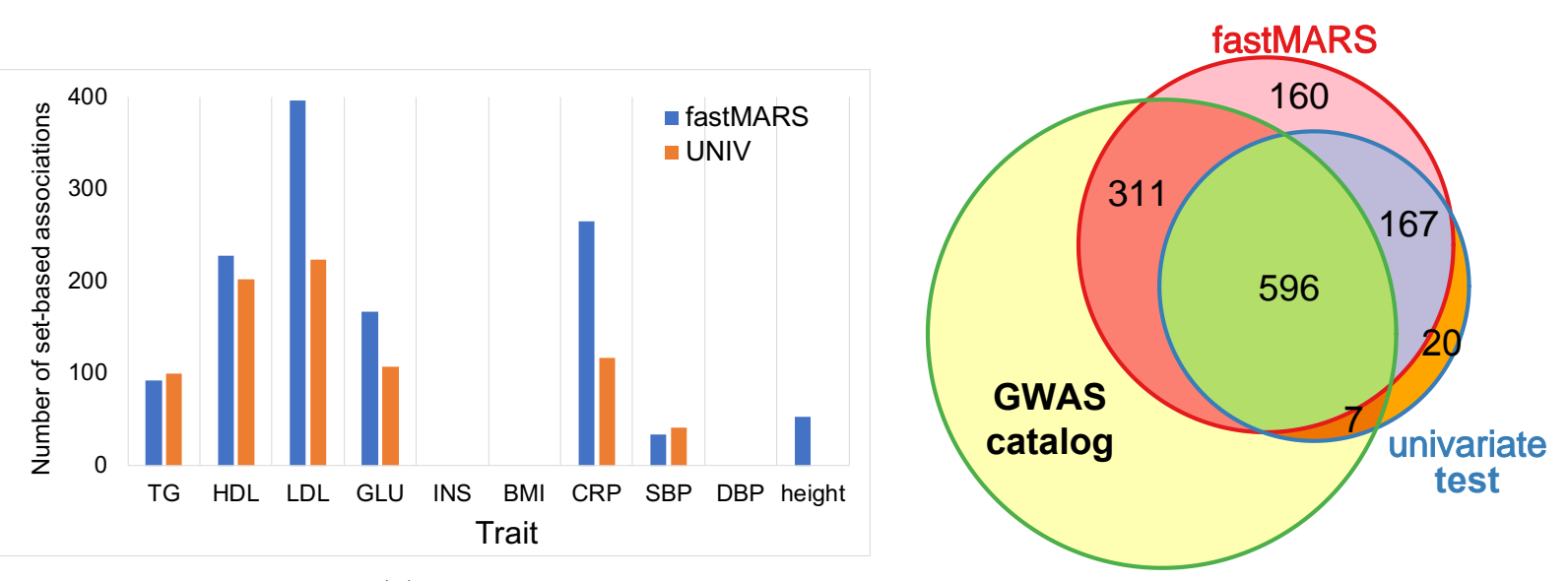

(a)

(b)

Figure 5: Significant associations identified by fastMARS and the univariate test in NFBC data (a) Number of significant associations identified by fastMARS and the univariate test. The $\mathrm{x}$-axis shows the 10 traits of NFBC data and the $\mathrm{y}$-axis shows the number of setbased associations that is likely to be associated with the traits. Blue bars show associations identified by fastMARS and orange bars show associations identified the univariate test. The 10 phenotypes are triglycerides (TG), high-density lipoproteins (HDL), low-density lipoproteins (LDL), glucose (GLU), insulin (INS), body mass index (BMI), C-reactive protein (CRP) as a measure of inflammation, systolic blood pressure (SBP), diastolic blood pressure (DBP), and height. (b) A Venn diagram showing the number of loci found by GWAS catalog for 10 traits. The red circle shows the number of loci identified by fastMARS, the blue circle shows the number of loci identified by the univariate test, and the green circle shows the number of identifications that were reported by GWAS catalog.

\section{Discussion}

Great efforts have been spent on finding the hidden heritability and many studies suspect that single level variant test misses signals due to small effect sizes and power problems. An approach that examines multiple variants together may increase statistical power to detect risk loci with small effect sizes. Moreover, interpreting Genome-Wide Association Studies (GWAS) at a gene level is an important step towards understanding the molecular processes that lead to disease [62, 63]. Several statistical approaches have been proposed that test the association between a set of variants and a trait or disease status, however, they simply use naive statistics such as a mean or sum of $\chi^{2}$ of statistics in the risk loci [23, 24, 64, 65].

Our method examines an association between a set of variants and a trait considering causal status and LD between the variants, utilizing the model used in one of the recent fine mapping approaches 
[13, 66]. Our method uses summary statistics from each variant, therefore, does not require raw data which is often unavailable publicly. Another main advantage of our method is that, for a locus, once a null panel of test statistics has been established, it could be applied to the locus on other studies. For example, in eQTL studies, once null panels of genes have been established, we can readily access significances of the genes in other tissues, and the same strategy can be applied to different traits in GWAS.

We applied our method to extensive simulated data sets with different effect sizes and the number of causal variants to show our method improves power compared to previous approaches including a widely used set-based association test, fastBAT, while successfully controls the type I error. Especially, we show that when there are many causal variants with small effect sizes, our method performs superior over the standard univariate association test approach. Applied to Genotype-Tissue Expression (GTEx) data, our method identifies more or comparable eGenes than the standard univariate approach as well as the ones reported by the GTEx consortium in all of the tissues. In addition, using the Whole Blood data, we show that a large portion of the eGenes identified only by MARS was reported by other larger studies as well as some of them have biological evidence of being eGenes based on many literatures. Lastly, utilizing Northern Finland Birth Cohort (NFBC) data, we show the effectiveness of our method on the GWAS that our method effectively identifies more association loci in GWAS compare to the standard association test approach.

We note some limitations of our work. First, MARS is computationally costly compared to the standard GWAS method as MARS test significance of an association based on re-sampling approach. However, in practice, we introduce fast and space efficient sampling techniques including importance sampling to reduce the sampling time dramatically, which well approximates the original result, while we were able to successfully handle big eQTL data sets containing tens of thousands of genes or GWAS data sets with thousands of samples. Second, we limited the number of causal variants in a locus to reduce the running time in the experiments. It is a reasonable assumption as previous studies have reported that a relatively small number of causal variants exist in a region. There is a trade-off between the number of causal variants to be considered and the running time, thus, to detect loci with many causal variants and raise the accuracy, one can increase the number of allowed causal variants, which can be set as an option in the program. Third, MARS can only be applied to common variants but not to rare variants as the MVN assumption holds only for common 
variants. Lastly, MARS does not utilize existing functional data as some of current methods have utilized functional data to detect more eGenes [67-69]. We can extend the statistical framework of MARS to utilize functional data for the future work. Despite these limitations, MARS is a novel statistical method that can detect newly associated loci and increase the number of loci that we can perform follow up studies. Through which, MARS can increase our biological understanding of diseases and complex traits.

\section{Materials and Methods}

\section{GWAS statistics}

Consider GWAS on a quantitative trait where we genotype $n$ individuals and collect a phenotype for the individuals. Let $X_{i}$ be a vector of length $n$ with the standardized genotypic values (i.e., mean zero and variance one) of $i$ th marker that we are testing and $Y$ be a vector of length $n$ with the phenotypic values. We assume that the data generating model follows the following linear additive model:

$$
Y=\mu \mathbf{1}_{\mathbf{n}}+X_{i} \beta_{i}+\mathbf{e}
$$

Here, $\mu$ is a mean of the phenotypic values, $\mathbf{1}_{\mathbf{n}}$ is a vector of $n$ ones, $\beta_{i}$ is their coefficients, and $\mathbf{e}$ is a vector of length $n$ sampled from $\mathcal{N}\left(0, \sigma^{2} \mathbf{I}\right)$ accounting for the residual errors, where $\mathbf{I}$ is an $n \times n$ identity matrix.

Under this model, the phenotype follows a MVN with a mean and variance as follows:

$$
Y \sim \mathcal{N}\left(\mu \mathbf{1}_{\mathbf{n}}+X_{i} \beta_{i}, \sigma_{e}^{2} \mathbf{I}\right)
$$

By maximizing likelihood of the model, we can estimate $\beta_{i}$ as follows:

$$
\hat{\beta}_{i}=\frac{X_{i}^{T} Y}{X_{i}^{T} X_{i}}, \hat{\beta}_{i} \sim \mathcal{N}\left(\beta_{i}, \frac{\sigma_{e}^{2}}{\left(X_{i}^{T} X\right)^{-1}}\right)
$$


and the summary statistic is computed as follows:

$$
S_{i}=\frac{\hat{\beta}_{i}}{\hat{\sigma}_{e}} \sqrt{X_{i}^{T} X_{i}}, S_{i} \sim \mathcal{N}\left(\lambda_{i}, 1\right)
$$

, where $\lambda_{i}$ is non-centrality parameter $(\mathrm{NCP})$ and is equal to $\frac{\beta_{i}}{\sigma_{e}} \sqrt{X_{i}^{T} X_{i}}$. We obtain the estimated values for $\mu$, e, and $\sigma_{e}$ as $\hat{\mu}=\frac{\mu \mathbf{1}_{\mathbf{n}}{ }^{T} X_{i}}{n}, \hat{e}=Y-\mathbf{1}_{\mathbf{n}} \hat{\mu}-\hat{\beta}_{i} X_{i}$, and $\hat{\sigma}_{e}=\sqrt{\frac{\hat{e}^{T} \hat{e}}{n-2}}$.

\section{The effect of Linkage Disequilibrium on the Statistics}

Consider the case that the $i$ th SNP is causal to a phenotype and the $j$ th SNP is non-causal but in LD with the $i$ th SNP. The correlation between the two variants is $r$, which is approximated by $\frac{1}{n} X_{j}^{T} X_{i}$. The effect size of the $j$ th SNP is computed as follows:

$$
\hat{\beta}_{j}=\frac{X_{j}^{T} Y}{X_{j}^{T} X_{j}}, \hat{\beta}_{j} \sim N\left(r \beta_{i}, \frac{\sigma_{e}^{2}}{\left(X_{j}^{T} X\right)^{-1}}\right)
$$

and the statistics for the $j$ th SNP is computed as follows:

$$
S_{j}=\frac{\hat{\beta}_{j}}{\hat{\sigma}_{e}} \sqrt{X_{j}^{T} X_{j}}, S_{j} \sim N\left(r \lambda_{i}, 1\right)
$$

We can show that the covariance between the statistics is equal to the correlation of the genotypes as follow:

$$
\begin{aligned}
\operatorname{Cov}\left(S_{i}, S_{j}\right) & =\frac{X_{i}^{T} X_{j}}{\sqrt{X_{i}^{T} X_{i}} \sqrt{X_{j}^{T} X_{j}}} \\
& =\operatorname{Cor}\left(X_{i}, X_{j}\right) \equiv r_{i j}
\end{aligned}
$$

Then, the joint distribution of the summary statistics for the two variants given their NCPs, $\lambda_{i}$ and $\lambda_{j}$, follows a multivariate normal distribution as follow:

$$
\left(\left[\begin{array}{l}
S_{i} \\
S_{j}
\end{array}\right] \mid\left[\begin{array}{l}
\lambda_{i} \\
\lambda_{j}
\end{array}\right]\right) \sim \mathcal{N}\left(\left[\begin{array}{l}
\lambda_{i} \\
\lambda_{j}
\end{array}\right],\left[\begin{array}{cc}
1 & r_{i j} \\
r_{i j} & 1
\end{array}\right]\right)
$$




\section{CAVIAR Generative Model}

Now we consider the case with $m$ SNPs. Given the true effect sizes of $m$ SNPs, $\Lambda=\left[\Lambda_{1}, \Lambda_{2}, \cdots, \Lambda_{m}\right]$, the summary statistics of $m$ SNPs, $S=\left[S_{1}, \cdots, S_{m}\right]^{T}$, follows:

$$
(S \mid \Lambda) \sim \mathcal{N}(\Sigma \Lambda, \Sigma)
$$

Here, $\Sigma$ is a correlation matrix, where $\Sigma\{i, j\}=r_{i j}$. We utilize Fisher's polygenic model and assume that effect sizes follow a normal distribution. Let $C$ be a binary vector of length $m$ that indicates the causal status of $m$ SNPs; 0 indicates a SNP is non-causal and 1 indicates a SNP is causal. Given a causal status $C$, we assume that the true effect size follows:

$$
(\Lambda \mid C) \sim \mathcal{N}(0, \Delta)
$$

, where $\Delta$ is a diagonal matrix with $\Delta\{i, i\}=\sigma^{2}$ if $i$ th SNP is causal and $\Delta\{i, j\}=\epsilon$, otherwise. From equation (1) and equation (2), the likelihood of summary statistics follows a multivariate normal distribution as follows:

$$
(S \mid C) \sim \mathcal{N}(0, \Sigma+\Sigma \Delta \Sigma)
$$

Then the likelihood function is given as follows:

$$
\mathcal{L}(\Sigma, \Delta \mid S)=\frac{1}{\sqrt{(2 \pi)^{m}|\Sigma+\Sigma \Delta \Sigma|}} \exp \left(-\frac{1}{2} S^{T}(\Sigma+\Sigma \Delta \Sigma)^{-1} S\right)
$$

We use a simple model that a probability of a SNP begin causal is $\gamma$, which is independent from other SNPs. Therefore, we compute the prior probability as follows:

$$
p(C)=\prod_{i=1}^{m} \gamma^{\left|c_{i}\right|}(1-\gamma)^{1-\left|c_{i}\right|}
$$

Here, $\left|c_{i}\right|=1$ if the $i$ th SNP is causal and $\left|c_{i}\right|=0$, otherwise. Although we use a simple prior, we can incorporate external information by using SNP-specific prior $\gamma_{i}$, which is the prior for the $i$ th SNP, then the prior probability to a more general case is $p\left(C \mid \gamma=\left[\gamma_{1}, \cdots \gamma_{m}\right]\right)=\prod_{i=1}^{m} \gamma_{i}^{\left|c_{i}\right|}\left(1-\gamma_{i}\right)^{1-\left|c_{i}\right|}$. 


\section{Model-based Association test Reflecting causal Status (MARS)}

MARS examines an association between a set of SNPs and a phenotype of interest. For the test statistic, we utilize a likelihood ratio test (LRT). We consider likelihoods of two models; the likelihood of the null model $\left(L_{0}\right)$ and the likelihood of the alternative model $\left(L_{1}\right)$. The null model assumes that there is no causal SNP to the phenotype and the alternative model assumes that there is at least one causal SNP for the phenotype. Then we can compute the test statistic as $L R T_{\text {score }}=L_{1} / L_{0}$. Given the observed marginal association statistics $S$ and correlation matrix $\Sigma$, we can compute the $L R T_{\text {score }}$ as follows:

$$
L R T_{\text {score }}=\frac{\sum_{C \in \zeta} p(S \mid C) p(C)}{p\left(S \mid C_{0}\right) p\left(C_{0}\right)}
$$

Here, we can compute the prior using equation (5) and the likelihood using equation (4). Since there are $m$ SNPs, there are $2^{m}$ possible causal statuses. In practice, we limit the number of allowed causal SNPs to 2 or 3 as which is consistent with reports from previous studies that a relatively small number of causal SNPs exist in a region. In addition, as the size of genes are often very big, e.g. many genes contain more than 10000 SNPs within $\pm 1 \mathrm{Mb}$ of TSS for the GTEx data, we order the SNPs by values of its summary statistics and used only top $50 \mathrm{SNPs}$ for computing the $L R T_{\text {scores }}$ to reduce the running time and the space. Figure6 (a) shows this practical implementation of MARS used for the experiments. This strategy reduces running time dramatically, while well approximates the results using all the SNPs in the loci (Supplementary Information 9) because the causal SNPs are expected to be included in the top 50 SNPs. In the case of limiting the number of causal SNPs up to 3 and using top 50 SNPs, there are $\sum_{i=1}^{3}\left(\begin{array}{c}50 \\ i\end{array}\right)$ casual statuses to be considered and $\zeta$ becomes a set that contains all the possible casual statuses with 1,2 , or 3 causal SNPs.

\section{eGene detection in GTEx data}

To identify an eGene, we examine the association between the gene expression levels and SNPs within $\pm 1 \mathrm{Mb}$ of TSS of the gene, which can be the candidates of cis-eQTLs for the gene. To assess the significance for a gene, we sample summary statistics from a MVN distribution under the null hypothesis, $S \sim N(0, \Sigma)$. Here, $\Sigma$ is a variance-covariance matrix estimated from the SNPs within 
$\pm 1 \mathrm{Mb}$ of TSS of the gene. We estimate the $L R T_{\text {scores }}$ for the null statistics to generate a null panel of $L R T_{\text {scores }} ; L R T_{\text {scores }}^{N U L L}$, using the equation(6). Then, we order the SNPs by their values of summary statistics and select top $50 \mathrm{SNPs}$ to compute the $L R T_{\text {score }}$ of the gene; $L R T_{\text {score }}^{D A T A}$, using the equation (6). The $p$-value of the gene is estimated as the quantile of $L R T_{\text {score }}^{D A T A}$ among $L R T_{\text {scores }}^{N U L}$ defined as follows:

$$
\frac{\text { Number of }\left(L R T_{\text {scores }}^{\text {DATA }}>L R T_{\text {scores }}^{\text {NULL }}\right)}{\text { Number of }\left(L R T_{\text {scores }}^{\text {NULL }}\right)}
$$

One of the advantages of MARS is that once the null panel, $L R T_{\text {scores }}^{N U L}$, has been estimated for a locus, the panel can be applied to the locus in any other tissues or traits rapidly to compute a $p$-value. We use the Whole Blood data, which contains the most number of samples among the 44 tissues, to estimate the null panels of 23163 genes and applied the panels to all the other tissues. The $p$-value threshold to identify eGenes is defined as the border of empirical $p$-values of eGene and non-eGene reported by GTEx consortium, which is differ by tissues as GTEx used FDR approach to find their eGenes. The similar process has been applied for detecting eGenes in the univariate test except for using maximum summary statistic as its test statistic instead of $L R T_{\text {score }}$.

\section{Power estimation}

To show MARS increases statistical power over the univariate test, we compare the power between MARS and the univariate test. For a fair comparison, we utilized the standard GWAS p-value threshold of $5 \times 10^{-8}$. We sample $10^{8}$ number of summary statistics under the null hypothesis, $S^{N U L L} \sim N(0, \Sigma)$, as well as $10^{8}$ number of summary statistics under the alternative hypothesis, $S^{A L T} \sim N(\Sigma \Lambda, \Sigma)$. Here, $\Lambda$ is a vector of length $m$, where $m$ is the number of SNPs, containing zeros except for the causal SNPs. For example, for a simulation, in which two SNPs, let's say SNP 1 and SNP 2, with effect size $\lambda$ is implanted in the data, $\Lambda$ is $[\lambda, \lambda, 0, \ldots, 0]$. We examined the power for cases with 2 causal or 3 causal SNPs implanted in the simulated data, where the causal SNPs are randomly selected for each simulation. Then, we compute the $p$-value of $S^{N U L L}$ using the univariate test; $\mathrm{UNI} p^{N U L L}$, and find the quantile $q$, where the $p$-value equals to the standard 
GWAS $p$-value threshold of $5 \times 10^{-8}$ as follows:

$$
q=\text { Number of }\left(\mathrm{UNI} p^{N U L L}<5 \times 10^{-8}\right)
$$

We compute the $L R T_{\text {scores }}$ of $S^{N U L L} ; L R T_{\text {scores }}^{N U L L}$, using MARS and set the $L R T_{\text {score }}$ at the quantile $q$ as the threshold of $L R T_{\text {scores }} ; L R T_{\text {score }}^{T H R}$, which satisfies the following equation:

$$
\text { Number of }\left(L R T_{\text {score }}^{N U L}>L R T_{\text {score }}^{T H R}\right)=q
$$

This $L R T_{\text {score }}^{T H R}$ corresponds to the standard GWAS $p$-value threshold of $5 \times 10^{-8}$. Now, we compute the $L R T_{\text {scores }}$ of $S^{A L T} ; L R T_{\text {scores }}^{A L T}$, and the power of MARS is defined as the number of $L R T_{\text {scores }}^{A L T}$ that is greater than the $L R T_{\text {score }}^{T H R}$ as follows:

$$
\text { Power of MARS }=\frac{\text { Number of }\left(L R T_{\text {score }}^{A L T}>L R T_{\text {score }}^{T H R}\right)}{10^{8}} \times 100
$$

The power of univariate test is defined similarly by computing the $p$-value of $S^{A L T}$ using the univariate test; UNI $p^{A L T}$, as follows:

$$
\text { Power of the univariate test }=\frac{\text { Number of }\left(\mathrm{UNI} p^{A L T}<5 \times 10^{-8}\right)}{10^{8}} \times 100
$$

In the power comparison of MARS and fastBAT, the power estimation process is the same as the one described above except for that $10^{5}$ simulations and a threshold of $10^{-5}$ is used instead of $10^{8}$ simulations and the threshold of $5 \times 10^{-8}$, accordingly. In the case, the univariate test is used to examine the quantile $q$, where the $p$-value of univariate test equals to $10^{-5}$. Then the quantile $q$ is used to find a threshold of MARS and that of fastBAT to compute the power of each method.

\section{Fast and space efficient sampling for MARS}

To access the significance of associations, MARS uses a re-sampling approach that requires a lot of sampling from MVN distribution. There are two main obstructions in this standard re-sampling approach. One is that a locus may contain many SNPs, for example, many genes in the GTEx data contain more than 10000 SNPs around $\pm 1 \mathrm{Mb}$ of their TSS. When the number of SNPs $m$ is very 
large, the standard re-sampling approach; $S \sim N\left(0, \Sigma_{m \times m}\right)$, using the Cholesky decomposition [70] is impractical. This not only takes a lot of time but also a lot of space as $\Sigma_{m \times m}$ itself often takes a few gigabytes of space. We reduce the space and time complexity dramatically utilizing the fact that $\Sigma_{m \times m}$ is a covariance matrix of $X ; \Sigma_{m \times m}=X^{T} X / n$, where $n$ is the number of samples. Instead of sampling statistics from MVN with the variance-covariance matrix of $\Sigma_{m \times m}$; $S \sim N\left(0, \Sigma_{m \times m}\right)$, we sample statistics from MVN with the variance-covariance matrix of $I_{n \times n} ;$ $S^{*} \sim N\left(0, I_{n \times n}\right)$. This neither takes time nor space because in general $n<<m$ and $n$ is not big. Then we multiply $S^{*}$ with $X^{T} / \sqrt{N}$ to compute the statistics $S=\frac{X^{T}}{\sqrt{N}} S^{*}$.

The other main obstruction of the standard re-sampling approach is that the number of sampling required to find a proper threshold for MARS may be very big. For the GTEx data, we compared the eGenes with those reported by the GTEx consortium and 10000 sampling was performed as which is the number of samples used for computing their empirical $p$-values. However, for the GWAS analysis, MARS needs to perform a lot of sampling to find a LRT threshold that corresponds to the standard GWAS $p$-value threshold of $5 \times 10^{-8}$. For the case, we apply importance sampling as follows. Instead of sampling from MVN with the variance-covariance matrix of $I_{n \times n}$; $S^{*} \sim N\left(0, I_{n \times n}\right)$, we sample statistics from MVN with the variance-covariance matrix of $\sqrt{2} I_{n \times n}$; $S_{i m p}^{*} \sim N\left(0, \sqrt{2} I_{n \times n}\right)$. Then the new statistics from importance sampling becomes $S_{i m p}=\frac{X^{T}}{\sqrt{N}} S_{i m p}^{*}$. We record an additional information, referred to as importance weight, defined as follows:

$$
W=\frac{f\left(S_{i m p}^{*} \mid 0, I_{n \times n}\right)}{f\left(S_{i m p}^{*} \mid 0, \sqrt{2} I_{n \times n}\right)}
$$

Here, $f$ indicates the probability density function of MVN. We repeat the process of sampling statistics $S_{i m p}^{*}$ and computing $S_{i m p}$ and $W, K$ times. Let's call each process as a run and after $K$ runs we have a set of statistics $\left\{S_{i m p_{1}}, S_{i m p_{2}}, \cdots, S_{i m p_{K}}\right\}$ and a set of weights $\left\{W_{1}, W_{2}, \cdots, W_{K}\right\}$. Now we estimate an univariate $p$-value from each $S_{i m p}$ and compute $p$-value threshold as the ratio of sum of weights that have the univariate $p$-value $<5 \times 10^{-8}$ over the sum of all the weights as follows:

$$
\frac{\sum_{i}^{C} W_{i}}{\sum_{i}^{K} W_{i}}
$$

Here, $i$ indicates the index of a run and $C$ is a set containing indices of runs with the univariate 
$p$-value $<5 \times 10^{-8}$. Given summary statistics of a locus, we access the significance of the locus by computing $L R T_{\text {score }}$ of the summary statistics; $L R T_{\text {score }}^{D A T A}$. We compute the $K$ number of $L R T_{\text {scores }}$ for the top 50 SNPs of the $S_{i m p} ; L R T_{\text {scores }}^{N U L L}$, as well. Then we compute the $p$-value of the locus as the ratio of sum of weights that have $L R T_{\text {scores }}^{N U L L}>L R T_{\text {scores }}^{D A T A}$ over the sum of all the weights as follows:

$$
\frac{\sum_{i}^{D} W_{i}}{\sum_{i}^{K} W_{i}}
$$

Here, $D$ is a set containing indices of runs with $L R T_{\text {scores }}^{N U L L}>L R T_{\text {scores }}^{D A T A}$. The association is significant if $p$-value estimated from the equation 8 is smaller than the $p$-value threshold estimated from the equation(7). Applied to randomly selected 10 genes, we find that the $p$-value estimated from $10^{4}$ number of importance sampling well approximates the $p$-values estimated from $10^{8}$ number of original re-sampling (Supplementary Information 5). Utilizing the importance sampling, we reduce the number of sampling dramatically from $10^{8}$ to $10^{4}$ on GWAS experiments. We refer to this fast and efficient version of MARS as fastMARS and Figure 6 shows the overview of association test for fastMARS (Figure 6(b)).

For the GTEx data analysis, we used MARS as described in Figure 6 (a), where $10^{4}$ number of sampling performed and upto 2 causal variants considered. In the case, MARS took approximately 3.5 minutes to test a significance for an average sized gene with 7522 SNPs for 338 samples in our system. Using parallel processing, we were able to run the 23163 number of genes in several hours; approximately 3 hours for sampling and computing $L R T_{\text {scores }}$ and some extra times for pre-processing and post-processing the data. For the NFBC data analysis, which used $10^{4}$ original sampling, we used fastMARS as described in 6 (b), where $10^{4}$ number of importance sampling performed and upto 2 causal variants considered. In the case, fastMARS took approximately 50 minutes to test a significance for an average sized locus with 299 SNPs for 5326 samples in our system. Using parallel processing, we were able to run the 56319 number of genes in approximately 2 days.

\section{The standard univariate test and fastBAT}

To compare MARS with a standard approach of set-based association test, we define an univariate test that uses a maximum summary statistic among the SNPs in the analysis locus. In addition, one 


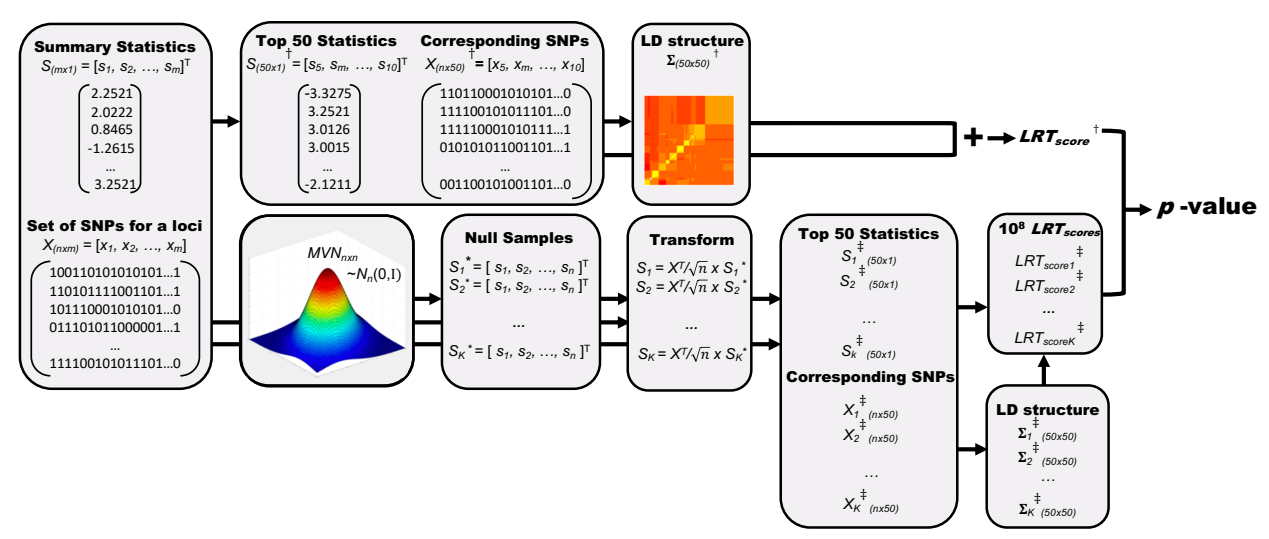

(a) Practical implementation of MARS

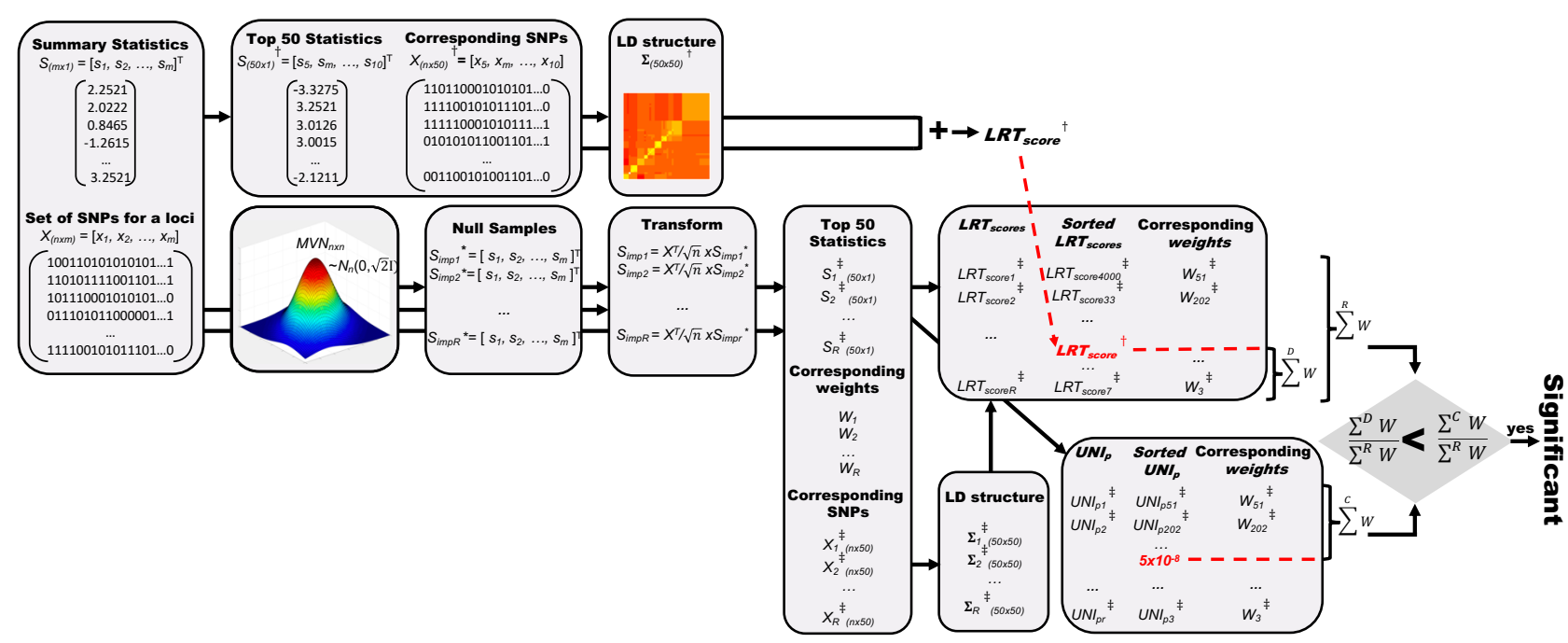

(b) Association test overview of fastMARS

Figure 6: (a) To reduce the running time and the space, MARS uses top 50 statistics instead of using all the SNPs in the analysis in practice. (b) For GWAS, we introduce fast and efficient sampling strategy.

of the widely used set-based association test, fastBAT (a fast and flexible set-Based Association Test using GWAS summary data) 24] is used for the comparison. GCTA (genome-wide complex trait analysis) [71] program was downloaded from the GCTA website (http://gcta.freeforums.net/thread/309/gctafastbat-based-association-analysis) and 'fastBAT' option was used for running the GCTA-fastBAT.

\section{GTEx data}

The summary statistics and genotypes of 44 tissues of GTEx data version 6 were downloaded from dbGap (https://www.ncbi.nlm.nih.gov/gap) and used to generate all the results throughout the paper. eGene list of GTEx data version 7 was download from dbGap as well and used only for the 
validation of eGenes that are identified by MARS applied on the GTEx data version 6. 23163 gene loci selected from the Whole Blood data was used for the analysis, which contain at least 50 SNPs in their $\pm 1 \mathrm{Mb}$ of TSS. We generate null panel of $L R T_{\text {scores }}$ using the Whole Blood data as which contains the most number of samples, 338. The numbers of genes are different between tissues due to the sample size differences and etc., thus, for the eGene detection in 44 tissues, we used gene regions common in each tissue and the Whole Blood data.

\section{Northern Finland Birth Cohort dataset}

The genotypes and 10 phenotype values of triglycerides (TG), high-density lipoproteins (HDL), low-density lipoproteins (LDL), glucose (GLU), insulin (INS), body mass index (BMI), C-reactive protein (CRP) as a measure of inflammation, systolic blood pressure (SBP), diastolic blood pressure (DBP), and height, over 5326 samples of the Northern Finland Birth Cohort (NFBC) dataset were downloaded from the dbGap. PLINK, a whole genome association analysis toolset (http://zzz.bwh.harvard.edu/pli is used to compute the statistics. For the set-based association test, gene map of the GTEx data, which contains 56319 gene positions, were used to define the loci to analyze. SNPs $\pm 1 \mathrm{Mb}$ around transcription start site (TSS) of the genes were searched in the NFBC genotype data and 51762 regions with more than 50 SNPs were used for the analysis. $10^{4}$ number of importance sampling were performed to generate null panel to estimate $p$-values of MARS and the univariate test.

\section{Software availability and license}

MARS software is implemented in $\mathrm{C}++$ and the program, source code, installation and running instructions are available at http://genetics.cs.ucla.edu/MARS/. MARS is offered under the GNU Affero GPL, Version 3 (AGPL-3.0). For details of the license, see https://www.gnu.org/licenses/ why-affero-gpl.html.

\section{Computing environment}

For the experiments we used UCLA Shared Hoffman2 Cluster, which currently consists of 1,200+ 64-bit nodes and 13,340 cores, with an aggregate of over 50TB of memory. Note that the cluster is shared by many users and each user has a limitation of 100 500 running jobs in par- 
allel, depends on the memory and the time that each job uses. The details are provided at https://www.hoffman2.idre.ucla.edu.

\section{Ethics approval}

No ethics approval was required for the study. 


\section{Acknowlogement}

This research was supported by Basic Science Research Program through the National Research Foundation of Korea(NRF) funded by the Ministry of Science, ICT \& Future Planning, No. 2017R1C1B5017497 and No. 2018M3E3A1057752. E.E. is supported by National Science Foundation grants 0513612, 0731455, 0729049, 0916676, 1065276, 1302448, 1320589, 1331176, and 1815624, and National Institutes of Health grants K25-HL080079, U01-DA024417, P01-HL30568, P01-HL28481, R01-GM083198, R01-ES021801, R01-MH101782,NIH BD2K award, U54EB020403 and R01-ES022282. We acknowledge the support of the NINDS Informatics Center for Neurogenetics and Neurogenomics (P30 NS062691). F.H. is supported by NIH T32 DK110919 and F32HG009987. 


\section{References}

1. Yang, J., Benyamin, B., McEvoy, B. P., Gordon, S., Henders, A. K., Nyholt, D. R., Madden, P. A., Heath, A. C., Martin, N. G., Montgomery, G. W., et al. (2010). Common SNPs explain a large proportion of the heritability for human height. Nat. Genet. 42, 565-569.

2. Wood, A. R., Esko, T., Yang, J., Vedantam, S., Pers, T. H., Gustafsson, S., Chu, A. Y., Estrada, K., Luan, J., Kutalik, Z., et al. (2014). Defining the role of common variation in the genomic and biological architecture of adult human height. Nat. Genet. 46, 1173-1186.

3. Goldstein, D. B. (2009). Common genetic variation and human traits. N. Engl. J. Med. 360, $1696-1698$.

4. Loh, P. R., Bhatia, G., Gusev, A., Finucane, H. K., Bulik-Sullivan, B. K., Pollack, S. J., de Candia, T. R., Lee, S. H., Wray, N. R., Kendler, K. S., et al. (2015). Contrasting genetic architectures of schizophrenia and other complex diseases using fast variance-components analysis. Nat. Genet. 47, 1385-1392.

5. Boyle, E. A., Li, Y. I., and Pritchard, J. K. (2017). An Expanded View of Complex Traits: From Polygenic to Omnigenic. Cell 169, 1177-1186.

6. Haiman, C. A., Le Marchand, L., Yamamato, J., Stram, D. O., Sheng, X., Kolonel, L. N., Wu, A. H., Reich, D., and Henderson, B. E. (2007). A common genetic risk factor for colorectal and prostate cancer. Nat. Genet. 39, 954-956.

7. Lango Allen, H., Estrada, K., Lettre, G., Berndt, S. I., Weedon, M. N., Rivadeneira, F., Willer, C. J., Jackson, A. U., Vedantam, S., Raychaudhuri, S., et al. (2010). Hundreds of variants clustered in genomic loci and biological pathways affect human height. Nature 467 , $832-838$.

8. Galarneau, G., Palmer, C. D., Sankaran, V. G., Orkin, S. H., Hirschhorn, J. N., and Lettre, G. (2010). Fine-mapping at three loci known to affect fetal hemoglobin levels explains additional genetic variation. Nat. Genet. 42, 1049-1051.

9. Stahl, E. A., Raychaudhuri, S., Remmers, E. F., Xie, G., Eyre, S., Thomson, B. P., Li, Y., 
Kurreeman, F. A., Zhernakova, A., Hinks, A., et al. (2010). Genome-wide association study meta-analysis identifies seven new rheumatoid arthritis risk loci. Nat. Genet. 42, 508-514.

10. Trynka, G., Hunt, K. A., Bockett, N. A., Romanos, J., Mistry, V., Szperl, A., Bakker, S. F., Bardella, M. T., Bhaw-Rosun, L., Castillejo, G., et al. (2011). Dense genotyping identifies and localizes multiple common and rare variant association signals in celiac disease. Nat. Genet. 43, 1193-1201.

11. Yang, J., Ferreira, T., Morris, A. P., Medland, S. E., Madden, P. A., Heath, A. C., Martin, N. G., Montgomery, G. W., Weedon, M. N., Loos, R. J., et al. (2012). Conditional and joint multiple-SNP analysis of GWAS summary statistics identifies additional variants influencing complex traits. Nat. Genet. 44, 369-375.

12. Flister, M. J., Tsaih, S.-W. W., O’Meara, C. C., Endres, B., Hoffman, M. J., Geurts, A. M., Dwinell, M. R., Lazar, J., Jacob, H. J., and Moreno, C. (2013). Identifying multiple causative genes at a single gwas locus. Genome Res 23, 1996-2002.

13. Hormozdiari, F., Kostem, E., Kang, E. Y., Pasaniuc, B., and Eskin, E. (2014). Identifying causal variants at loci with multiple signals of association. Genetics 198, 497-508.

14. Kichaev, G., Yang, W. Y., Lindstrom, S., Hormozdiari, F., Eskin, E., Price, A. L., Kraft, P., and Pasaniuc, B. (2014). Integrating functional data to prioritize causal variants in statistical fine-mapping studies. PLoS Genet. 10, e1004722.

15. Hormozdiari, F., Kichaev, G., Yang, W. Y., Pasaniuc, B., and Eskin, E. (2015). Identification of causal genes for complex traits. Bioinformatics 31, i206-213.

16. Hormozdiari, F., Zhu, A., Kichaev, G., Ju, C. J., Segre, A. V., Joo, J. W. J., Won, H., Sankararaman, S., Pasaniuc, B., Shifman, S., et al. (2017). Widespread Allelic Heterogeneity in Complex Traits. Am. J. Hum. Genet. 100, 789-802.

17. Jansen, R., Hottenga, J. J., Nivard, M. G., Abdellaoui, A., Laport, B., de Geus, E. J., Wright, F. A., Penninx, B. W. J. H., and Boomsma, D. I. (2017). Conditional eQTL analysis reveals allelic heterogeneity of gene expression. Hum. Mol. Genet. 26, 1444-1451. 
18. Consortium, I. H. (2003). The international hapmap project. Nature 426, 789-96.

19. Frazer, K. A., Ballinger, D. G., Cox, D. R., Hinds, D. A., Stuve, L. L., Gibbs, R. A., Belmont, J. W., Boudreau, A., Hardenbol, P., Leal, S. M., et al. (2007). A second generation human haplotype map of over 3.1 million SNPs. Nature 449, 851-861.

20. Abecasis, G. R., Altshuler, D., Auton, A., Brooks, L. D., Durbin, R. M., Gibbs, R. A., Hurles, M. E., McVean, G. A., Altshuler, D., Durbin, R. M., et al. (2010). A map of human genome variation from population-scale sequencing. Nature 467, 1061-1073.

21. Li, M. X., Gui, H. S., Kwan, J. S., and Sham, P. C. (2011). GATES: a rapid and powerful gene-based association test using extended Simes procedure. Am. J. Hum. Genet. 88, 283293.

22. Sul, J. H., Han, B., and Eskin, E. (2011). Increasing power of groupwise association test with likelihood ratio test. J. Comput. Biol. 18, 1611-1624.

23. Ionita-Laza, I., Lee, S., Makarov, V., Buxbaum, J. D., and Lin, X. (2013). Sequence kernel association tests for the combined effect of rare and common variants. Am. J. Hum. Genet. 92, 841-853.

24. Bakshi, A., Zhu, Z., Vinkhuyzen, A. A., Hill, W. D., McRae, A. F., Visscher, P. M., and Yang, J. (2016). Fast set-based association analysis using summary data from GWAS identifies novel gene loci for human complex traits. Sci Rep 6, 32894.

25. Consortium, G. (2015). Human genomics. the genotype-tissue expression (gtex) pilot analysis: multitissue gene regulation in humans. Science 348, 648-60.

26. Battle, A., Brown, C. D., Engelhardt, B. E., Montgomery, S. B., Aguet, F., Ardlie, K. G., Cummings, B. B., Gelfand, E. T., Getz, G., Hadley, K., et al. (2017). Genetic effects on gene expression across human tissues. Nature 550, 204-213.

27. Joehanes, R., Zhang, X., Huan, T., Yao, C., Ying, S. X., Nguyen, Q. T., Demirkale, C. Y., Feolo, M. L., Sharopova, N. R., Sturcke, A., et al. (2017). Integrated genome-wide analysis of expression quantitative trait loci aids interpretation of genomic association studies. Genome Biol. 18, 16. 
28. Sawcer, S., Hellenthal, G., Pirinen, M., Spencer, C. C., Patsopoulos, N. A., Moutsianas, L., Dilthey, A., Su, Z., Freeman, C., Hunt, S. E., et al. (2011). Genetic risk and a primary role for cell-mediated immune mechanisms in multiple sclerosis. Nature 476, 214-219.

29. Speedy, H. E., Di Bernardo, M. C., Sava, G. P., Dyer, M. J., Holroyd, A., Wang, Y., Sunter, N. J., Mansouri, L., Juliusson, G., Smedby, K. E., et al. (2014). A genome-wide association study identifies multiple susceptibility loci for chronic lymphocytic leukemia. Nat. Genet. $46,56-60$.

30. Sille, F. C., Thomas, R., Smith, M. T., Conde, L., and Skibola, C. F. (2012). Post-GWAS functional characterization of susceptibility variants for chronic lymphocytic leukemia. PLoS ONE 7, e29632.

31. Matesanz, F., Potenciano, V., Fedetz, M., Ramos-Mozo, P., Abad-Grau, M. d. e. 1. M., Karaky, M., Barrionuevo, C., Izquierdo, G., Ruiz-Pena, J. L., Garcia-Sanchez, M. I., et al. (2015). A functional variant that affects exon-skipping and protein expression of SP140 as genetic mechanism predisposing to multiple sclerosis. Hum. Mol. Genet. 24, 5619-5627.

32. He, P., Xia, W., Wang, L., Wu, J., Guo, Y. F., Zeng, K. Q., Wang, M. J., Bing, P. F., Xie, F. F., Lu, X., et al. (2018). Identification of expression quantitative trait loci (eQTLs) in human peripheral blood mononuclear cells (PBMCs) and shared with liver and brain. J. Cell. Biochem. 119, 1659-1669.

33. Sun, W., Kechris, K., Jacobson, S., Drummond, M. B., Hawkins, G. A., Yang, J., Chen, T. H., Quibrera, P. M., Anderson, W., Barr, R. G., et al. (2016). Common Genetic Polymorphisms Influence Blood Biomarker Measurements in COPD. PLoS Genet. 12, e1006011.

34. Thalayasingam, N., Nair, N., Skelton, A. J., Massey, J., Anderson, A. E., Clark, A. D., Diboll, J., Lendrem, D. W., Reynard, L. N., Cordell, H. J., et al. (2018). CD4+ and B Lymphocyte Expression Quantitative Traits at Rheumatoid Arthritis Risk Loci in Patients With Untreated Early Arthritis: Implications for Causal Gene Identification. Arthritis Rheumatol 70, 361-370.

35. Sabatti, C., Service, S. K., Hartikainen, A.-L. L., Pouta, A., Ripatti, S., Brodsky, J., Jones, 
C. G., Zaitlen, N. A., Varilo, T., Kaakinen, M., et al. (2009). Genome-wide association analysis of metabolic traits in a birth cohort from a founder population. Nat Genet 41, $35-46$.

36. MacArthur, J., Bowler, E., Cerezo, M., Gil, L., Hall, P., Hastings, E., Junkins, H., McMahon, A., Milano, A., Morales, J., et al. (2017). The new NHGRI-EBI Catalog of published genomewide association studies (GWAS Catalog). Nucleic Acids Res. 45, D896-D901.

37. Lu, X., Huang, J., Mo, Z., He, J., Wang, L., Yang, X., Tan, A., Chen, S., Chen, J., Gu, C. C., et al. (2016). Genetic Susceptibility to Lipid Levels and Lipid Change Over Time and Risk of Incident Hyperlipidemia in Chinese Populations. Circ Cardiovasc Genet 9, 37-44.

38. Coram, M. A., Duan, Q., Hoffmann, T. J., Thornton, T., Knowles, J. W., Johnson, N. A., Ochs-Balcom, H. M., Donlon, T. A., Martin, L. W., Eaton, C. B., et al. (2013). Genome-wide characterization of shared and distinct genetic components that influence blood lipid levels in ethnically diverse human populations. Am. J. Hum. Genet. 92, 904-916.

39. Mora, S. and Ridker, P. M. (2006). Justification for the Use of Statins in Primary Prevention: an Intervention Trial Evaluating Rosuvastatin (JUPITER)-can C-reactive protein be used to target statin therapy in primary prevention? Am. J. Cardiol. 97, 33A-41A.

40. Kathiresan, S., Melander, O., Guiducci, C., Surti, A., Burtt, N. P., Rieder, M. J., Cooper, G. M., Roos, C., Voight, B. F., Havulinna, A. S., et al. (2008). Six new loci associated with blood low-density lipoprotein cholesterol, high-density lipoprotein cholesterol or triglycerides in humans. Nat. Genet. 40, 189-197.

41. Soranzo, N., Rivadeneira, F., Chinappen-Horsley, U., Malkina, I., Richards, J. B., Hammond, N., Stolk, L., Nica, A., Inouye, M., Hofman, A., et al. (2009). Meta-analysis of genome-wide scans for human adult stature identifies novel Loci and associations with measures of skeletal frame size. PLoS Genet. 5, e1000445.

42. Gudbjartsson, D. F., Walters, G. B., Thorleifsson, G., Stefansson, H., Halldorsson, B. V., Zusmanovich, P., Sulem, P., Thorlacius, S., Gylfason, A., Steinberg, S., et al. (2008). Many sequence variants affecting diversity of adult human height. Nat. Genet. 40, 609-615. 
43. Weedon, M. N., Lango, H., Lindgren, C. M., Wallace, C., Evans, D. M., Mangino, M., Freathy, R. M., Perry, J. R., Stevens, S., Hall, A. S., et al. (2008). Genome-wide association analysis identifies 20 loci that influence adult height. Nat. Genet. 40, 575-583.

44. Sanna, S., Jackson, A. U., Nagaraja, R., Willer, C. J., Chen, W. M., Bonnycastle, L. L., Shen, H., Timpson, N., Lettre, G., Usala, G., et al. (2008). Common variants in the GDF5-UQCC region are associated with variation in human height. Nat. Genet. 40, 198-203.

45. Lettre, G., Jackson, A. U., Gieger, C., Schumacher, F. R., Berndt, S. I., Sanna, S., Eyheramendy, S., Voight, B. F., Butler, J. L., Guiducci, C., et al. (2008). Identification of ten loci associated with height highlights new biological pathways in human growth. Nat. Genet. 40, $584-591$.

46. He, M., Xu, M., Zhang, B., Liang, J., Chen, P., Lee, J. Y., Johnson, T. A., Li, H., Yang, X., Dai, J., et al. (2015). Meta-analysis of genome-wide association studies of adult height in East Asians identifies 17 novel loci. Hum. Mol. Genet. 24, 1791-1800.

47. Wood, A. R., Esko, T., Yang, J., Vedantam, S., Pers, T. H., Gustafsson, S., Chu, A. Y., Estrada, K., Luan, J., Kutalik, Z., et al. (2014). Defining the role of common variation in the genomic and biological architecture of adult human height. Nat. Genet. 46, 1173-1186.

48. Berndt, S. I., Gustafsson, S., Magi, R., Ganna, A., Wheeler, E., Feitosa, M. F., Justice, A. E., Monda, K. L., Croteau-Chonka, D. C., Day, F. R., et al. (2013). Genome-wide metaanalysis identifies 11 new loci for anthropometric traits and provides insights into genetic architecture. Nat. Genet. 45, 501-512.

49. Kanai, M., Akiyama, M., Takahashi, A., Matoba, N., Momozawa, Y., Ikeda, M., Iwata, N., Ikegawa, S., Hirata, M., Matsuda, K., et al. (2018). Genetic analysis of quantitative traits in the Japanese population links cell types to complex human diseases. Nat. Genet. 50, 390-400.

50. Lettre, G., Palmer, C. D., Young, T., Ejebe, K. G., Allayee, H., Benjamin, E. J., Bennett, F., Bowden, D. W., Chakravarti, A., Dreisbach, A., et al. (2011). Genome-wide association 
study of coronary heart disease and its risk factors in 8,090 African Americans: the NHLBI CARe Project. PLoS Genet. 7, e1001300.

51. Willer, C. J., Sanna, S., Jackson, A. U., Scuteri, A., Bonnycastle, L. L., Clarke, R., Heath, S. C., Timpson, N. J., Najjar, S. S., Stringham, H. M., et al. (2008). Newly identified loci that influence lipid concentrations and risk of coronary artery disease. Nat. Genet. 40, 161-169.

52. Aulchenko, Y. S., Ripatti, S., Lindqvist, I., Boomsma, D., Heid, I. M., Pramstaller, P. P., Penninx, B. W., Janssens, A. C., Wilson, J. F., Spector, T., et al. (2009). Loci influencing lipid levels and coronary heart disease risk in 16 European population cohorts. Nat. Genet. $41,47-55$.

53. Davis, J. P., Huyghe, J. R., Locke, A. E., Jackson, A. U., Sim, X., Stringham, H. M., Teslovich, T. M., Welch, R. P., Fuchsberger, C., Narisu, N., et al. (2017). Common, lowfrequency, and rare genetic variants associated with lipoprotein subclasses and triglyceride measures in Finnish men from the METSIM study. PLoS Genet. 13, e1007079.

54. Surakka, I., Horikoshi, M., Magi, R., Sarin, A. P., Mahajan, A., Lagou, V., Marullo, L., Ferreira, T., Miraglio, B., Timonen, S., et al. (2015). The impact of low-frequency and rare variants on lipid levels. Nat. Genet. 47, 589-597.

55. Kathiresan, S., Willer, C. J., Peloso, G. M., Demissie, S., Musunuru, K., Schadt, E. E., Kaplan, L., Bennett, D., Li, Y., Tanaka, T., et al. (2009). Common variants at 30 loci contribute to polygenic dyslipidemia. Nat. Genet. 41, 56-65.

56. Willer, C. J., Schmidt, E. M., Sengupta, S., Peloso, G. M., Gustafsson, S., Kanoni, S., Ganna, A., Chen, J., Buchkovich, M. L., Mora, S., et al. (2013). Discovery and refinement of loci associated with lipid levels. Nat. Genet. 45, 1274-1283.

57. Nagy, R., Boutin, T. S., Marten, J., Huffman, J. E., Kerr, S. M., Campbell, A., Evenden, L., Gibson, J., Amador, C., Howard, D. M., et al. (2017). Exploration of haplotype research consortium imputation for genome-wide association studies in 20,032 Generation Scotland participants. Genome Med 9, 23. 
58. Waterworth, D. M., Ricketts, S. L., Song, K., Chen, L., Zhao, J. H., Ripatti, S., Aulchenko, Y. S., Zhang, W., Yuan, X., Lim, N., et al. (2010). Genetic variants influencing circulating lipid levels and risk of coronary artery disease. Arterioscler. Thromb. Vasc. Biol. 30, 22642276.

59. Spracklen, C. N., Chen, P., Kim, Y. J., Wang, X., Cai, H., Li, S., Long, J., Wu, Y., Wang, Y. X., Takeuchi, F., et al. (2017). Association analyses of East Asian individuals and transancestry analyses with European individuals reveal new loci associated with cholesterol and triglyceride levels. Hum. Mol. Genet. 26, 1770-1784.

60. Teslovich, T. M., Musunuru, K., Smith, A. V., Edmondson, A. C., Stylianou, I. M., Koseki, M., Pirruccello, J. P., Ripatti, S., Chasman, D. I., Willer, C. J., et al. (2010). Biological, clinical and population relevance of 95 loci for blood lipids. Nature 466, 707-713.

61. Ligthart, S., Vaez, A., Hsu, Y. H., Stolk, R., Uitterlinden, A. G., Hofman, A., Alizadeh, B. Z., Franco, O. H., and Dehghan, A. (2016). Bivariate genome-wide association study identifies novel pleiotropic loci for lipids and inflammation. BMC Genomics 17, 443.

62. Botstein, D. and Risch, N. (2003). Discovering genotypes underlying human phenotypes: past successes for mendelian disease, future approaches for complex disease. Nat. Genet. 33 Suppl, 228-237.

63. Kann, M. G. (2010). Advances in translational bioinformatics: computational approaches for the hunting of disease genes. Brief. Bioinformatics 11, 96-110.

64. Liu, J. Z., McRae, A. F., Nyholt, D. R., Medland, S. E., Wray, N. R., Brown, K. M., Hayward, N. K., Montgomery, G. W., Visscher, P. M., Martin, N. G., et al. (2010). A versatile genebased test for genome-wide association studies. Am. J. Hum. Genet. 87, 139-145.

65. Li, M. X., Kwan, J. S., and Sham, P. C. (2012). HYST: a hybrid set-based test for genomewide association studies, with application to protein-protein interaction-based association analysis. Am. J. Hum. Genet. 91, 478-488.

66. Hormozdiari, F., van de Bunt, M., Segre, A. V., Li, X., Joo, J. W. J., Bilow, M., Sul, J. H., 
Sankararaman, S., Pasaniuc, B., and Eskin, E. (2016). Colocalization of GWAS and eQTL Signals Detects Target Genes. Am. J. Hum. Genet. 99, 1245-1260.

67. Eskin, E. (2008). Increasing power in association studies by using linkage disequilibrium structure and molecular function as prior information. Genome Res. 18, 653-660.

68. Wen, X. (2016). Molecular qtl discovery incorporating genomic annotations using bayesian false discovery rate control. The Annals of Applied Statistics 10, 1619-1638.

69. Duong, D., Zou, J., Hormozdiari, F., Sul, J. H., Ernst, J., Han, B., and Eskin, E. (2016). Using genomic annotations increases statistical power to detect eGenes. Bioinformatics 32, i156-i163.

70. Hajivassiliou, V., McFadden, D., and Ruud, P. (1996). Simulation of multivariate normal rectangle probabilities and their derivatives theoretical and computational results. Journal of Econometrics 72, 85-134.

71. Yang, J., Lee, S. H., Goddard, M. E., and Visscher, P. M. (2011). GCTA: a tool for genome-wide complex trait analysis. Am. J. Hum. Genet. 88, 76-82. 